\title{
PHOTOELECTRON ENERGY LOSSES TO THERMAL ELECTRONS
}

\author{
ROBERT W. SCHUNK* and PAUL B. HAYS \\ The University of Michigan, Ann Arbor, Mich. 48105, U.S.A.
}

(Recelved in final form 7 September 1970)

\begin{abstract}
The photoelectron energy loss rate to the ambient electrons is discussed. It is shown that the conventional Butler and Buckingham expression has been incorrectly applied in photoelectron calculations. The appropriate energy loss rates are indicated. It is also shown that the geomagnetic field has little influence on the photoelectron energy loss rate.
\end{abstract}

\section{INTRODUCTION}

The calculation of the photoelectron energy degradation in a planetary atmosphere requires a knowledge of the rate of energy loss to the ambient electrons. Most of the theoretical calculations of the photoelectron energy distribution to date have used an expression derived by Butler and Buckingham (1962). A few widely quoted examples are Dalgarno, McElroy and Moffett (1963), Hanson (1963), Geisler and Bowhill (1965), Hoegy, Fournier and Fontheim (1965), Nisbet (1968) and Fontheim, Beutler and Nagy (1968). This expression, derived by considering two-body Coulomb encounters, neglects the generation of plasma waves. Furthermore, in these studies the close collision theory has itself been incorrectly applied. This note summarizes recent results which can be used to evaluate the energy loss rate of a photoelectron which has a velocity much greater than the thermal speed. It is shown that the correct application of the test particle energy loss rate theory can increase the loss by as much as 70 per cent over that currently used. It is also shown that in the Earth's atmosphere the ambient magnetic field has very little influence on the rate of energy loss of a photoelectron.

\section{THE DEGRADATION OF PHOTOELECTRON ENERGY}

Perkins (1965) and Itikawa and Aono (1966) have discussed the rate of energy loss of a test particle in a thermal plasma. For photoelectrons, where the test particle velocity is greater than ion and electron thermal velocities, the loss of energy to ambient ions is negligible and the loss rate can be expressed as

$$
-\frac{\mathrm{d} E}{\mathrm{~d} t}=\frac{\omega_{p}^{2} e^{2}}{v}\left\{J_{e}\left(\frac{v}{v_{e}}\right) \ln \frac{2 \Lambda}{3 \gamma}+\frac{v}{v_{s}} G_{e}\left(\frac{v}{v_{e}}\right)\right\}
$$

where

$$
J_{e}(u)=\frac{2}{\sqrt{\pi}}\left\{\int_{0}^{u} e^{-x^{2}} \mathrm{~d} x-2 u e^{-u^{2}}\right\}
$$

and

$$
\Lambda=\frac{3(k T)^{3 / 2}}{e^{3}(4 \pi n)^{1 / 2}}, \quad \omega_{p}=\left(\frac{4 \pi n e^{2}}{m}\right)^{1 / 2}
$$

(cf. Itikawa and Aono, 1966). Here the first term in the brackets is the expression commonly used to describe the photoelectron energy loss rate (Butler and Buckingham, 1962; Dalgarno, McElroy and Moffett, 1963; Hanson, 1963). In these expressions $\omega_{p}$ is the electron

*I.S.T. Postdoctoral Fellow. 
plasma frequency, $m$ the electron mass, $n$ the electron density, $T$ the electron temperature, $k$ Boltzmann's constant, $v$ the test electron speed, $E$ the energy, $v_{e}=(2 k T / m)^{1 / 2}$ the electron thermal speed, $-e$ the electron charge and $\ln \gamma$ is Euler's constant. The term of primary interest here, $G_{e}\left(v / v_{e}\right)$, represents the dependence of the Coulomb logarithm on the photoelectron velocity and contains the energy loss rate due to the Cerenkov emission of plasma waves. For a singly-charged ion values of $G(u)$ are given by Itikawa and Aono (1966) for all values of $u$. However, for an electron $G_{e}(u)$ is known only for certain values of $u$. In order to facilitate the explanation of the results to follow, we will describe expression (1) in its entirety rather than $G_{e}(u)$ alone. Consider the following limiting regions:

(a) High speed photoelectron (classical treatment) $k T \ll E \ll m e^{4} / 2 \hbar^{2}$

In the velocity range $v / v_{e} \gg 1$, Equation (1) reduces to an expression given by Kihara and Aono (1963)

$$
-\frac{\mathrm{d} E}{\mathrm{~d} t}=\frac{\omega_{p}^{2} e^{2}}{v} \ln \frac{m v^{3}}{\gamma e^{2} \omega_{p}}
$$

The separate effects of Coulomb collisions and plasma wave generation can be seen by writing (4) in the form,

$$
-\frac{\mathrm{d} E}{\mathrm{~d} t}=\frac{\omega_{p}^{2} e^{2}}{v}\left\{\ln \left(\frac{m v^{2} l_{d}}{2 e^{2}}\right)+\ln \left(\frac{v}{l_{d} \omega_{p}}\right)+\ln \left(\frac{2}{\gamma}\right)\right\}
$$

where $l_{d}=\left(k T / 4 \pi n e^{2}\right)^{1 / 2}$ is the electron Debye length.

The first term in (5) is the energy loss due to two-body Coulomb collisions and corresponds to the asymptotic Butler and Buckingham (1962) expression. The argument in the logarithm is the ratio of the Debye length to the distance of closest approach of the photoelectron and a stationary field electron. We note here the principal error in the previous photoelectron applications. If one replaces the distance of closest approach between the photoelectron and a thermal electron by that for two thermal electrons, one obtains the common $\ln \Lambda$ dependence given by Dalgarno, McElroy and Moffett (1963). For a high speed photoelectron the correct distance of closest approach can be considerably smaller than that for two thermal electrons. We note also that this collisional logarithm is not correct when the de Broglie wavelength of the photoelectron becomes comparable with the distance of closest approach.

The second term in (5) accounts for the energy loss due to the Cerenkov emission of plasma waves, a term mentioned but neglected by Butler and Buckingham (1962). This term was first derived by Pines and Bohm (1952) and is indeed important for fast particles. We note that in the complete formulation including both two-body and collective encounters the Debye cut-off length does not appear in the combined result. This is to be expected, since the concept of a Debye length cut-off is entirely artificial for fast encounters.

The third term in expression (5) indicates that the energy loss due to two-body encounters can be separated from that due to the radiation of plasma waves only with logarithmic accuracy.

(b) High speed photoelectron (quantum mechanical treatment) $E \gg m e^{4} / 2 \hbar^{2}$

It was noted in the last section that the classical treatment would allow the distance of closest approach between particles to become smaller than the de Broglie wavelength of the photoelectron. This, however, cannot occur in an actual collision. Thus, in the region where 
the de Broglie wavelength is greater than the classical distance of closest approach,

or in terms of the energy where

$$
\lambda=\frac{\hbar}{\mu g}>\frac{e^{2}}{\mu g^{2}}
$$

$$
E>\frac{m e^{4}}{2 \hbar^{2}} \approx 14 \mathrm{eV} \text { for electrons }
$$

one must use a quantum mechanical treatment for close encounters. Here, $2 \pi \hbar$ is Planck's constant, $g$ is the relative velocity and $\mu$ is the reduced mass equal to $m / 2$ for electrons. We note here that the characteristic length for wave generation is the Debye length. Since this length is much greater than the de Broglie wavelength, the plasma wave term will not be modified in the quantum limit. One then finds that the previous formula can be approximately corrected by replacing the distance of closest approach by the de Broglie wavelength. However, this is only good to logarithmic accuracy, and neglects the influence of exchange for identical particles. Larkin (1960) has treated this problem by an exact formulation and obtains an asymptotic expression for $E \gg m e^{4} / 2 \hbar^{2}$ by using the Green's function method and diagram technique. He finds that

$$
-\frac{\mathrm{d} E}{\mathrm{~d} t}=\frac{\omega_{\nu}^{2} e^{2}}{v}\left[\ln \frac{m v^{2}}{\hbar \omega_{\nu}}-0.04\right]
$$

where the last term in the brackets represents the influence of exchange and is negligible.

(c) The influence of magnetic fields

During the investigation of the influence of the Cerenkov emission of plasma waves one further question was considered. That is, what is the influence of the additional modes of wave propagation which occur when a magnetic field exists in a plasma? This effect has been investigated by Honda, Aono and Kihara (1963) in the classical limit and by Akhiezer (1961) in the quantum limit. These authors show that in the classical limit

and in the quantum limit

$$
-\frac{\mathrm{d} E}{\mathrm{~d} t}=\frac{\omega_{p}^{2} e^{2}}{v}\left[\ln \frac{m v^{3}}{\gamma e^{2} \omega_{p}}-f\right]
$$

$$
-\frac{\mathrm{d} E}{\mathrm{~d} t}=\frac{\omega_{p}^{2} e^{2}}{v}\left[\ln \frac{m v^{2}}{\hbar \omega_{p}}-f\right]
$$

where the function $f$ contains the effect of the magnetic field and is a function of the electron cyclotron frequency, $\omega_{b}$, and the pitch angle of the photoelectron. The function $f$ is the same in both cases and for ionospheric applications where $\omega_{b} / \omega_{p} \sim 1$ one finds that $f<0.5$. Thus, compared to the logarithmic terms, which are of order 20 , the influence of the magnetic field is negligible.

\section{SUMMARY}

The energy loss rate of an energetic photoelectron $(E \gg k T)$ is independent of the electron temperature and can be described approximately by the formula

$$
-\frac{d E}{\mathrm{~d} t}=\frac{\omega_{p}^{2} e^{2}}{v} \begin{cases}\ln \left(\frac{m v^{3}}{\gamma e^{2} \omega_{p}}\right) & \text { for } \quad k T \ll E<\frac{m e^{4}}{2 \hbar^{2}} \\ \ln \left(\frac{m v^{2}}{\hbar \omega_{p}}\right) & \text { for } E>\frac{m e^{4}}{2 \hbar^{2}}\end{cases}
$$


where there is a slight uncertainty in the energy range where the distance of closest approach calculated by the classical theory is equal to the de Broglie wavelength of the photoelectron in the center of mass system. These expressions are compared, in Fig. 1, with the energy loss rate used in most of the present photoelectron calculations. We note the existence of an error in the previous calculations of electron-electron energy loss rates of as much as 70 per cent. An error of this magnitude, however, will probably not markedly alter the predicted heating of the ionosphere because of the relative energy and altitude variations of electronelectron and electron-neutral cross sections.

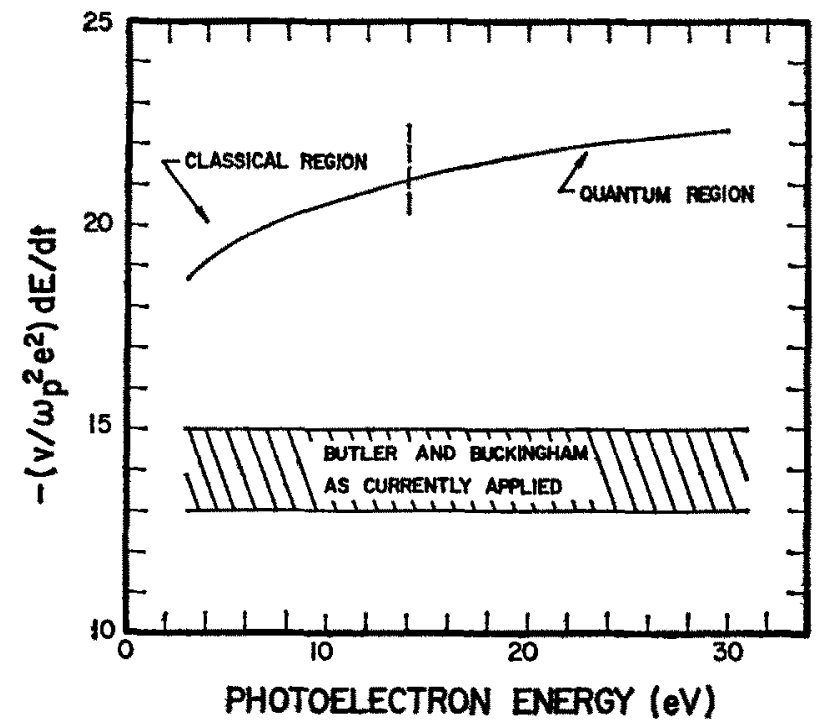

Fig. 1. Comparison of Photoelectron energy loss rates. For this comparison $n=10^{6} \mathrm{~cm}^{-\mathrm{x}}$.

It is also noted that the influence of the ionospheric magnetic field is negligible in the calculation of photoelectron energy loss rates.

Acknowledgement-This research was supported in part by NASA Grant 23-005-360.

\section{REFERENCES}

AKriezier, J. A. (1961). Theory of the interaction of a charged particle with a plasma in a magmetic field. Soviet Phys. JETP 13, 667-672.

Butler, S. T. and Buckingham, M. J. (1962). Energy loss of a fast ion in a plasma. Phys. Rev. 126, 1-4.

Dalgarno, A., McElroy, M. B. and Moffett, R. J. (1963). Electron temperatures in the ionosphere. Planet. Space Sci. 11, 463-484.

Fontherm, E. G., Beutler, A. E. and NaGY, A. F. (1968). Theoretical calculations of the conjugate predawn effects. Annls Géophys. 24, 489.

GEISLER, J. E. and Bowinl, S. A. (1965). Energy exchange between the ionosphere and the protonosphere. J. atmos. terr. Phys. 27, 1119-1146.

Hanson, W. B. (1963). Electron temperatures in the upper atmosphere. Space Research III, pp. 282-302. North-Holland, Amsterdam.

HoEgY, W. R., Fournier, J. P. and Fontheim, E. G. (1965). Photoelectron energy distribution in the $F$ region. J. geophys. Res. 70, 5464-5468.

Honda, N., AONo, O. and KIhara, T. (1963). Fluctuations in a plasma III. Effect of the magnetic field on the stopping power. J. phys. Soc. Japan 18, 256-260. 
ITIKAWA, Y. and AONO, O. (1966). Energy change of a charged particle moving in a plasma. Physics Fluids 9, 1259-1261.

KmaRA, T. and Aono, O. (1963). Unified theory of relaxations in plasmas, I. Basic theorem. J. phys. Soc. Japan 18, 837-851.

LARKIN, A. I. (1960). Passage of particles through a plasma. Soviet Phys. JETP 10, 186-191.

NISBET, J. S. (1968). Photoelectron escape from the ionosphere. J. atmos. terr. Phys. 30, 1257-1278.

PINEs, D. and BOHM, D. (1952). A collective description of electron interactions: II. Collective vs. individual particle aspects of the interactions. Phys. Rev. 85, 338-353.

Perkrns, F. (1965). Motion of a test particle in a plasma. Physics Fluids 8, 1361-1367. 